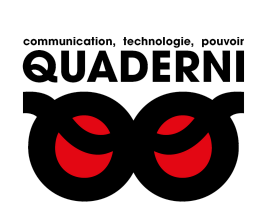

\title{
Quaderni
}

Communication, technologies, pouvoir

95 | Hiver 2017-2018

Logiques numériques des radicalisations

\section{Le conspirationnisme 2.0}

Entretien avec Raphaël Josset et Rudy Reichstadt

Raphaël Josset, Rudy Reichstadt et Emmanuel Taïeb

\section{(2) OpenEdition}

Journals

Édition électronique

URL : https://journals.openedition.org/quaderni/1146

DOI : 10.4000 /quaderni. 1146

ISSN : 2105-2956

Éditeur

Les éditions de la Maison des sciences de l'Homme

Édition imprimée

Date de publication : 5 février 2018

Pagination : 79-94

Référence électronique

Raphaël Josset, Rudy Reichstadt et Emmanuel Taïeb, « Le conspirationnisme 2.0 », Quaderni [En ligne], 95 | Hiver 2017-2018, mis en ligne le 05 février 2020, consulté le 03 septembre 2021. URL : http:// journals.openedition.org/quaderni/1146; DOI : https://doi.org/10.4000/quaderni. 1146 


\section{Politique}

\section{Le conspirationnisme 2.0}

\section{Entretien avec Raphaël Josset et Rudy Reichstadt}

Cet entretien est la version remaniée d'une émission de radio, "La Valeur de l'Homme", proposée et présentée par Emmanuel Taïeb, qui a été diffusée sur Fréquence Protestante le 26 mai 2017. Sa retranscription a été faite avec l'aimable autorisation de la radio, et revue par les auteurs.

Emmanuel Taïeb : Bonjour à tous et toutes, très heureux de vous retrouver en direct pour ce Midi Magazine. L'émission d'aujourd'hui a pour titre « Le conspirationnisme 2.0 ». Le conspirationnisme est « à la mode ». Il ne se passe pas une journée sans que les réseaux sociaux ne proposent une interprétation complotiste des événements

Docteur en sociologie Université Paris-Descartes

\section{Rudy Reichstadt}

Président de l'Observatoire du conspirationnisme

\section{par Emmanuel Taïeb}

Institut Universitaire de France Sciences Po Lyon, Triangle du moment. Récemment, le célèbre basketteur Shaquille O'Neal se faisait l'écho des théories fumeuses de la terre plate, en signalant que lorsqu'il traverse les États-Unis, il voit bien que la route est plate et donc la terre n'est pas sphérique. Il a depuis déclaré que tout cela était une plaisanterie ${ }^{1}$, mais les théories dites « platistes » ont connu grâce à lui un large écho ${ }^{2}$. D'autres théories du complot connaissent une fortune ininterrompue : le complot juif et les Protocoles des Sages de Sion ${ }^{3}$, les complots des élites ou des banquiers ${ }^{4}$, le complot des Illuminati ${ }^{5}$, qui rencontre un succès étonnant chez les jeunes et dans la culture populaire $^{6}$, le complot des agences internationales pour dominer le monde ${ }^{7}$, ou encore le complot des gouvernements qui organiseraient des attentats sur leur propre territoire pour faire porter le chapeau à la minorité musulmane dans sa version radicalisée. Cet imaginaire du complot est désormais très balisé, et de nombreux travaux 
de diverses disciplines universitaires l'ont bien cerné notamment dans sa dimension politique, car les idées complotistes ne sont pas simplement des récits fantaisistes ou étranges, elles sont avant tout des discours politiques et idéologiques qui proposent une vision toute faite du monde, et qui entendent peser sur le jeu politique national ${ }^{8}$. Il y a donc un militantisme complotiste qui utilise les médias numériques pour concurrencer l'information professionnelle et pour gagner chaque jour de nouveaux adeptes au milieu de la désinformation et des « fake news » qui abondent sur les réseaux sociaux. Le conspirationnisme a su renouveler sa petite musique pessimiste pour devenir un phénomène 2.0 à part entière.

Mes invités sont Raphaël Josset, docteur en sociologie de l'Université Paris-Descartes et chercheur au Centre d'études sur l'actuel et le quotidien. Il est spécialiste des mutations socioanthropologiques des sociétés, et a publié récemment un ouvrage intitulé Complosphère. L'esprit conspirationniste à l'ère des réseaux 9 . Et Rudy Reichstadt, qui anime depuis bientôt dix ans l'Observatoire du conspirationnisme (Conspiracy Watch $^{10}$ ), site web très riche sur les « théories » du complot, avec de nombreux articles de divers contributeurs, proposant une veille engagée et des analyses du phénomène complotiste.

Emmanuel Taïeb : Je voudrais que l'on commence par une définition, ou en tout cas une approche, de ce phénomène conspirationniste, complotiste, ou de ce phénomène des « théories » du complot. On le définit habituellement comme l'idée ou la croyance que le cours des événements est nécessairement voulu par des gens, par un groupe caché d'hommes qui tirent les ficelles.
Que pourrait-on ajouter pour cerner un peu mieux ce phénomène ? Est-ce que l'on pourrait dire que c'est une espèce de « contre-récit » systématique des événements, des contre-propositions, la proposition d'une autre réalité ? Et comment peut-on approcher de ce phénomène ?

Raphaël Josset : Pour ma part, je l'ai abordé par rapport à l'imaginaire du grand complot mondial. C'est une vision très particulière, basée sur l'idée qu'il y a un complot global. Il y a forcément du complot dans le monde, ça aussi il faut le mettre en avant, il faut en parler, mais là on traite - en tout cas moi quand je traite du conspirationnisme -, je traite de l'idée que pour les complotistes il existerait un groupe en particulier qui tire les ficelles au niveau mondial, et tous les événements, tout ce qui arrive dans le monde serait le fruit d'une planification de ces gens-là. Selon les acteurs de cette mouvance, les responsables ont différents noms, donc chacun va en adopter un, soit les extra-terrestres, soit les Illuminati, comme vous l'avez dit, soit les francs-maçons, etc. Effectivement, ce sont des contre-propositions aussi, ce sont d'autres visions du monde, ce sont d'autres « vérités » finalement qui se confrontent à d'autres visions du monde, mais voilà, là c'est une vision conspirationniste du monde où tout s'explique par le complot, par un grand complot, par un plan secret dont le but serait l'établissement de la domination totale d'une élite d'initiés maléfiques. C'est une vision très particulière...

Emmanuel Taïeb : Mais ce qui vous a intéressé, Raphaël Josset, c'est de dire : il y a un imaginaire du complot qui se déploie régulièrement et qui est un imaginaire d'explication, d'accusation, mais 
ce qui vous intéressait au fond c'est la manière dont cet imaginaire est constitué. À quel type de vision du monde renvoie-t-il ?

Raphaël Josset : Oui, moi ce qui m'intéressait, en particulier, ce n'était pas de polémiquer sur les faits, à savoir si telle chose est arrivée ou pas, s'il $\mathrm{y}$ a réellement telle chose cachée derrière ceci ou cela, si untel existe. Sinon on n'en finit plus. On perd notre temps à essayer de convaincre par des arguments logiques, rationnels, des gens qui de toute façon ne veulent pas savoir, ne veulent pas forcément entendre ces choses-là.

Moi, ce n'est pas ce qui m'a intéressé, ce qui m'a intéressé, c'est le schéma de pensée, c'est ce qui advient systématiquement, c'est la structure qui se dégage dans ce mode de représentation des choses. En l'occurrence, l'idée obsessionnelle que le bien et le mal s'affrontent, qu'il y a un petit groupe de gens qui agissent dans l'ombre, dans les ténèbres, donc ce sont les forces du mal. On a une espèce de retour d'une pensée théologique pour le coup, et dont j'essaie de faire un peu la généalogie dans le livre. C'est une théologie qui remonte peut-être au fondement des dualismes dans la sphère indo-européenne autour du mazdéisme indo-iranien et plus spécifiquement de la réforme de celui-ci par le prophète Zarathoustra qui va systématiser la vision du combat entre le dieu de la Lumière essentielle et les maléfiques forces des Ténèbres. Un combat organisant l'histoire des hommes et qui doit se terminer par la destruction des trompeuses forces du mal après la venue d'un Sauveur messie-rédempteur. Après ça se décline dans toute la pensée dualiste de l'Occident, dans toute la métaphysique occidentale. Ça a structuré nos représentations et orienté nos comportements. Ça se retrouve de manière presque caricaturale chez les conspirationnistes chez qui c'est très tranché, c'est très « manichéen » : simplement cette idée d'opposition entre le bien et le mal, qu'il y a deux pôles qui s'affrontent, et que là ce sont forcément les forces du mal qui agissent cachées dans l'ombre.

C'est toujours ce schéma qui revient systématiquement, donc moi j'utilise Gilbert Durand ${ }^{11}$, je parle des «structures anthropologiques de l'imaginaire » qui mettent en avant deux régimes dans leur représentation de la conscience. Gilbert Durand parlait du régime « diurne » et du régime « nocturne » de l'imaginaire. Ce qui domine effectivement dans la pensée conspirationniste et qui a longtemps dominé aussi d'une manière générale en Occident, c'est le régime diurne, la " structure héroïque » et/ou " schizomorphe », c'est l'idée du héros solaire qui représente la lumière, qui va combattre les forces ténébreuses du mal tapies dans l'ombre. Ça peut représenter plein de choses et on trouve cette mise en scène dans une multitude de mythologies sur la planète mais elle est tout à fait caractéristique de la vision conspirationniste du monde obsédée par l'archétype de l'Ombre.

Emmanuel Taïeb : Donc réactivation des strates de ce dualisme entre le bien et le mal : le conspirationnisme fonctionne dans une désignation d'un mal que l'on ne verrait pas, dans une désignation de l'ennemi. C'est peut-être ce qui le rend à la fois fort, et un peu inquiétant quand on travaille dessus, car le conspirationnisme est une accusation.

Rudy Reichstadt : C'est une accusation, c'est pour ça que c'est un discours préoccupant, 
d'ailleurs. Léon Poliakov parlait de causalité diabolique $^{12}$, et on retrouve cette structure simplificatrice, manichéenne - le Bien, le Mal - dans le discours conspirationniste. C'est dire aussi qu'il est en partie lié, ce discours, avec une forme de paresse intellectuelle. Évidemment, le monde est plus complexe que la manière dont les complotistes se le représentent.

Raphaël Josset a eu raison de rappeler au début de son propos qu'il y a des complots bien sûr. C'est important de dissiper ce malentendu qui est présent à chaque fois qu'on entreprend en fait de critiquer les thèses conspirationnistes. On s'entend répondre presque systématiquement : « mais alors, pour vous, les complots n'existent pas? ». Évidemment que non. Nous, ce qui nous intéresse, ce sont les complots imaginaires, les croyances erronées ou extravagantes dans des complots qui n'existent pas. Évidemment, à côté de ça, l'histoire est faite aussi bien entendu de complots. Il y a des complots, il y en aura encore tant qu'il y aura des sociétés humaines, du secret et de la politique en général. Mais dire que l'histoire est faite de complots ne doit pas occulter le fait que l'histoire est faite aussi de croyances en tout genre, de mythes, et aussi de mythes complotistes.

Et ces mythes complotistes ont parfois eu des conséquences, et, plus souvent qu'à leur tour, des conséquences absolument funestes ; et je parle là du complotisme en tant que discours qui vient accompagner un passage à l'acte violent, un processus de persécutions, voire un passage à l'acte criminel, génocidaire. On sait que le complotisme anti-Juif ou anti-Tutsis a préparé des génocides, littéralement, et qu'aujourd'hui le complotisme est au cœur de l'idéologie djihadiste qui motive le passage à l'acte terroriste. D'ailleurs, ce complotisme est également utilisé pour recruter et inciter les gens à partir en Syrie faire le djihad. Donc voilà, ce ne sont pas juste des « petites histoires drôles ».

Maintenant, sur la définition du conspirationnisme : il y a à peu près autant de définitions que d'auteurs sur le sujet. On peut l'aborder évidemment comme une forme de contre-culture. En général, je l'aborde sous la forme d'un discours sans exclusive, par ce que c'est un discours qui est très plastique, qui peut être mis au service d'idéologies politiques tout à fait opposées, tout à fait rivales. C'est pour ça d'ailleurs que même s'il y a des liens très forts historiquement entre antisémitisme et conspirationnisme, tout conspirationnisme n'est pas par définition antisémite. Il y a un conspirationnisme dans la droite israélienne la plus extrême par exemple. Vous avez un conspirationnisme qui n'a rien d'antisémite, mais qui va par exemple expliquer que Yitzhak Rabin a été assassiné par Shimon Peres. Ce sont des thèses qui ont cours en Israël, en tout cas dans une frange de l'extrême-droite religieuse très marquée. Cela permet de rappeler que personne n'a l'exclusivité du conspirationnisme.

Emmanuel Taïeb : Raphaël Josset, sur la question de la mise en accusation, vous indiquez dans votre ouvrage qu'il y a un «potentiel totalitaire $~^{13}$ des théories du complot, qu'elles peuvent se transformer en une espèce de procès en sorcellerie. Que dans cette vision du monde, il y a des catégorisations et qu'on va catégoriser un certain nombre de groupes, d'acteurs, d'institutions du mauvais côté de la barrière. Est-ce bien l'idée? 
Raphaël Josset : Oui, c'est la logique du bouc émissaire, donc on va designer tel ou tel groupe social ou telle personne comme responsable de tous les malheurs du monde. On va désigner ce qui est passé dans le langage populaire comme le « bouc émissaire », et qui aussi est une pratique. On s'est déchargé du mal en sacrifiant un bouc et là, c'est un peu la même chose, on désigne l'ennemi comme ça, et qui en plus risque de n'être qu'un ennemi imaginaire. En tout cas, on rend responsable des gens de choses dont ils ne sont pas forcément responsables, et on veut s'en débarrasser. Tout ça peut amener éventuellement et précisément à des logiques génocidaires, où on va essayer d'éradiquer le mal en exterminant les gens. C'est ça le danger, sinon on ne s'y intéresserait pas. Ça peut amener à cette extrémité éventuellement, et donc ce n'est pas juste pour discuter de cette chose-là, ce n'est pas juste distrayant, ça peut l'être par ailleurs - parfois c'est assez drôle -, mais ça peut être aussi assez dangereux.

Ça suit une mauvaise pente, c'est une espèce de logique inquisitoriale, où l'on va chercher les déviants, les hérétiques, ceux qui ne sont pas dans la norme et donc éventuellement c'est la chasse aux sorcières, les suppôts de Satan, comme il y a eu les grandes chasses aux sorcières par le passé. Mais le souci justement, c'est que - quand nous traitons de ce problème-là - il ne faut pas que l'on tombe nous-mêmes dans la même logique, et c'est ce qui arrive dès que l'on commence à designer des individus sur le mode «oui, toi tu es conspirationniste et c'est pas bien! ». Le problème c'est ça, c'est comment traitons-nous de cet objet sans tomber dans le même processus inquisitorial en stigmatisant les gens comme ça, en leur collant une étiquette sur le front, en mettant en avant une espèce de nébuleuse que l'on va considérer négativement comme étant « obscurantiste »? C'était en tout cas mon approche, mon souci au départ.

Rudy Reichstadt : Je crois qu'à ce stade, il est important de distinguer ce que l'on entend derrière le mot conspirationnisme. Qu'est-ce que le « conspirationnisme » ? Ça peut être plusieurs choses en réalité. Ça peut être un théoricien du complot ou un désinformateur professionnel, comme il en existe sur le marché de l'information, sur Internet, et qui peut générer un véritable business. Alex Jones, aux États-Unis, qui est un peu le « pape » du conspirationnisme américain, a un chiffre d'affaires annuel estimé à plus de 10 millions de dollars par an. C'est assez considérable. Alain Soral, qui est quelqu'un qui se revendique du «national-socialisme », qui a été condamné plusieurs fois pour antisémitisme, et qui fait un usage abusif et répété de la théorie du complot, revendique une trentaine de salariés. Donc, il y a un vrai business du complotisme, même si je pense fondamentalement que le motif est avant tout idéologico-politique.

On ne peut évidemment pas mettre dans le même sac des désinformateurs professionnels comme ces gens-là, et des gens qui sont séduits par ces discours-là, et pour qui rien n'est jamais forcément joué. C'est-à-dire des personnes que l'on peut toujours ramener vers plus de raison en essayant de les exposer aussi à des contrepoints critiques de ces théories du complot, si les gens ne sont pas de mauvaise foi. Après tout, on peut très bien être intoxiqué par ces contenus-là et revenir vers peut-être plus de raison face à des arguments. 
C'est pour ça qu'à mon avis c'est très important - et c'est ce que j'essaie de faire - de répondre aux arguments complotistes. Pas à tous, mais en tout cas à ceux qui sont les plus influents, les plus présents sur le «marché cognitif», pour parler comme Gérald Bronner. Parce qu'être ébranlé par un argument complotiste ça n'a rien d'illégitime en soi, ça nous arrive, ça arrive à chacun de nous.

Emmanuel Taïeb : Ça renvoie à du doute, finalement.

Rudy Reichstadt : Bien sûr, mais là où les journalistes et la société civile ont une responsabilité, c'est qu'à un moment il faut aussi essayer de répondre à ces arguments, les critiquer, les démonter, et faire la part des choses entre le vrai et le faux ou l'invérifiable.

Emmanuel Taïeb : On le disait tout à l'heure : les gens, qui travaillent sur le complotisme, ne nient pas l'existence des complots. Ce qui les intéressent au fond c'est le «-isme ». Ce qui est intéressant, c'est cette dimension politique ou idéologique. C'est-à-dire qu'il y a du point de vue de ceux qui produisent ces théories - qui sont les désinformateurs ou les agitateurs - une vraie volonté d'occuper un terrain idéologique, dont l'un des leviers est le discours conspirationniste, parce que c'est un discours un peu inquiétant qui marche. Est-ce qu'il n'y a pas aussi cette dimension extrêmement politique et idéologique?

Rudy Reichstadt : Mais bien sûr, elle est même essentielle, Richard Hofstadter qui est un des pionniers de la réflexion sur le sujet parlait de style paranoïaque ${ }^{14}$ - on ne parlait pas encore de conspirationnisme dans les années 1960 -, et soulignait bien qu'il y a une différence fondamentale entre la paranoïa clinique qui relève de la psychopathologie, et le style paranoïaque, le conspirationnisme, qui est une forme de paranoïa politique. Donc il faut effectivement « repolitiser» le sujet, pour parler comme vous, Emmanuel Taïeb ${ }^{15}$.

Il faut d'autant plus le faire que l'on sait aujourd'hui, grâce à la psychologie sociale notamment, qu'il y a un lien net, une corrélation, entre adhésions à des théories du complot et extrémismes politiques et choix de l'extrémisme ${ }^{16}$. Pourquoi ? Parce que - et déjà Hofstadter l'expliquait - lorsqu' on vous raconte matin, midi et soir que vos représentants démocra-tiquement élus sont en réalité des pantins à la solde de puissances obscures, que la démocratie est un vaste théâtre d'ombres, et que les choses se décident ailleurs, eh bien vous en êtes fatalement, logiquement, conduit à remettre en cause la validité même du système qui produit cela, qui rend possible cela, et donc la démocratie libérale, la société ouverte. Cette affinité élective qui peut exister entre l'extrémisme, la radicalité politique d'une part, le conspirationnisme de l'autre, me paraît importante à souligner.

Emmanuel Taïeb : Raphaël Josset, sur cette vision d'un pouvoir derrière le pouvoir, d'un vrai pouvoir qui tirerait les ficelles derrière le pouvoir démocratique, c'est ça le « régime nocturne », c'est cet imaginaire-là qui est activé dans finalement une forme de réenchantement du monde, mais un réenchantement négatif, un réenchantement pessimiste? 
Raphaël Josset : C'est ça, c'est une espèce de réenchantement négatif effectivement. Ce qui met en avant le fait qu'on est confronté à un désenchantement, un désenchantement du monde, une " démagification », comme disait Max Weber. C'est quelque chose qu'il faut traiter aussi. Ce sont aussi des leitmotivs et des problématiques de mon livre : penser le conspirationnisme par ce biais-là, et poser la question de savoir de quoi il est le signe. C'est un symptôme, il y a ça qui émerge, c'est une espèce de tendance lourde, c'est monté en puissance depuis le début du XXI ${ }^{\mathrm{e}}$ siècle, et ça devient quelque chose de complètement viral, il contamine tous les esprits, et après ça peut être un mouvement de pensée comme un autre. Mais qu'est-ce qui fait qu'il y a là une tendance que l'on peut très bien observer? De quoi cela est-ce le signe? Qu'est-ce que ça montre?

Pour moi c'est le signe d'une mutation: on cherche à retrouver une explication, un sens du monde, un sens à la vie, en général. Donc, c'est le retour d'un grand récit qui par ailleurs reprend les grands schèmes des récits apocalyptiques, tels qu'on les trouve dans la Bible et généralement c'est quelque chose qui est largement mis en avant. C'est Norman Cohn qui parlait des "fanatiques de l'apocalypse », en tout cas dans la traduction française de son livre sur les mouvements millénaristes au Moyen Âge ${ }^{17}$, et lui-même après a écrit sur le mythe du complot juif et la redécouverte des Protocoles des Sages de Sion, et toute l'enquête qu'il y a eu pour prouver que c'était un faux ${ }^{18}$. En tout cas, il y a une corrélation entre la pensée apocalyptique et la pensée conspirationniste. C'est aussi le signe d'une espèce de crise existentielle et spirituelle, une crise de civilisation qui est très profonde, et c'est une manière d'y remédier, comme il y a plein d'autres choses aussi qui émergent pour essayer de remédier à cette crise-là, à cet effondrement de la présence comme le disait l'anthropologue italien Ernesto de Martino ${ }^{19}$, dont je parle un peu dans mon livre.

C'est quelque chose de fondamental cette question de la présence à soi, aux autres, au monde qui s'effondre. On est maintenant dans un monde de plus en plus spectral, fantomatique, de plus en plus virtuel, de plus en plus dans l'ère de la simulation à la Baudrillard. C'est peut-être ça le monde fantomatique, dont parlait par ailleurs Günther Anders. Anders, dès les années 1950, avait eu une réflexion intéressante par rapport à ça en parlant justement de la radio, de la télévision, des médias. Il disait que le monde devenait de plus en plus fantomatique, donc qu'il y avait une espèce de présence, comme nos voix [à la radio], une présence un petit peu partout, mais en même temps c'est une absence, c'est fantomatique, parce que nous ne sommes pas vraiment là physiquement. Mais il y a quand même une présence, qui est cette espèce de spectre, et le monde devient comme ça finalement, et donc on peut toujours imaginer des choses. Cette idée du spectre qui hante le monde, ça remagifie, ça réenchante un petit peu, mais ce n'est pas merveilleux, c'est un peu glauque, c'est un peu triste.

\section{Emmanuel Taïeb : Inquiétant?}

Raphaël Josset : Oui, c'est dans l'angoisse, ça montre une angoisse existentielle.

Emmanuel Taïeb : Mais est-ce que c'est lié aussi au fait que finalement ce que l'on a ap- 
pelé le désenchantement du monde, le discours scientifique auquel les systèmes occidentaux, démocratiques, sont très attachés, ce discours scientifique a été incapable de prendre en charge au fond les destinées individuelles ou la demande de spiritualité ? Est-ce qu'il y aurait quelque chose de cet ordre-là ? L'idée qu'au fond la science n'a pas été capable de remplir le vide laissé par le désenchantement du monde?

Raphaël Josset : Ce que disait Max Weber, c'est que l'avènement du rationalisme, de la rationalité, a désenchanté le monde. L'avènement de la science fait progresser des choses, c'est sûr, mais ça a détruit d'autres choses. C'est ce que l'on appelle le désenchantement, la crise des valeurs, et le déclin des idées religieuses, des croyances, du sacré, de la spiritualité. On arrive dans un monde de causalités mécaniques, purement matérielles. C'est toute l'opposition entre matérialisme et spiritualité. On est dans l'avènement de cette espèce d'ère du vide et de la science, qui peut être aussi un nouveau récit à un moment donné, un nouveau récit qui...

Emmanuel Taïeb : ...marque ses limites finalement?

Raphaël Josset : Voilà. Il ne suffit pas à apporter suffisamment d'enthousiasme, finalement. Ce n'est pas tellement la science elle-même, son récit, son discours, qui pourraient fonctionner, mais ses applications technologiques qui ont bouleversé nos rapports au monde, à l'espace et au temps, et qui constamment produisent de la mutation, donc de l'impermanence, de la précarité, de l'instabilité. Nous sommes confrontés tout le temps à des choses qui changent nos vies, et c'est difficile à vivre.

Rudy Reichstadt : On est vraiment dans un âge post-positiviste, c'est-à-dire que l'idée selon laquelle « la Science » allait tout régler - qui est très dix-neuviémiste - est morte et enterrée. Le $\mathrm{XX}^{\mathrm{e}}$ siècle a été le moment de la rupture de confiance avec les promesses de la science, parce qu'il y a eu Hiroshima et Tchernobyl. Il faut bien avoir à l'esprit que parmi les trois grandes instances d'autorité, les trois grandes paroles d'autorité aujourd'hui contestées, notamment à travers le conspirationnisme, il y a la parole politique de l'État en général, mais politique en général, la parole des médias, la presse, et puis la parole scientifique. La science est vraiment mise à mal à travers le complotisme. On remet en doute des faits scientifiques comme l'exploration de la Lune. On remet en cause aussi le réchauffement climatique. Un président américain a été élu bien qu'il ait pu déclarer que le réchauffement climatique était une intox créée «par et pour les Chinois »! Ça va quand même très loin le conspirationnisme contemporain.

Je crois que fondamentalement le complotisme donne l'illusion d'une maîtrise sur quelque chose d'absolument immaitrisable. Face à un phénomène comme celui de la mondialisation, qui est un phénomène un peu homéostatique, que personne ne dirige, même s'il y a des instances de concertation internationales - le G7, le G20 qui essaient de réguler -, l'idée du complot mondial, des Illuminati, des « sionistes », des « reptiliens », place un pilote dans l'avion. Quelque part c'est rassurant, on se dit que finalement, on sait où on va quand même... 
Emmanuel Taïeb : Rudy Reichstadt, vous écrivez dans un de vos textes ${ }^{20}$ "la montée en puissance d'Internet a considérablement changé la donne. Internet s'est en effet accompagné d'une propagation inédite du conspirationnisme dans le débat public, s'imposant au cours des quinze dernières années comme un "contreespace public » nimbé de la légitimité de la vox populi et se définissant en rupture avec les médias traditionnels. ». Quelle est cette idée d'un «contre-espace public»?

Rudy Reichstadt : Dans l'espace public « légitime », celui de la presse classique, le rapport de force est évidemment en faveur des anti-complotistes. On pensera par exemple aux initiatives de « fact checking » du Monde ou de Libération. En revanche, sur Internet ces choses-là sont inversées, c'est-à-dire que même s'ils ne sont sans doute pas plus nombreux, les internautes complotistes sont en revanche beaucoup plus actifs. Ils font beaucoup plus de bruit, et par conséquent ils saturent Internet, ou en tout cas ils colonisent avec leurs thèses ce continent vierge qu'est Internet, ce continent cognitif informationnel. Tout simplement parce qu'ils sont plus motivés que les autres, que les indifférents, pour produire des théories du complot. Internet fournit une prime au complotisme, à la croyance en général, mais à la croyance complotiste en particulier parce qu'il donne un écho aux plus motivés. Internet, c'est la loi du plus fort.

Quand, dans les années 1980 ou 1990, vous vous intéressiez au trésor des Templiers par exemple, vous pouviez trouver à la bibliothèque du coin ou en librairie des ouvrages sur le sujet, mais ça s'arrêtait là. C'était compliqué de creuser.
Aujourd'hui, non seulement vous allez trouver cent fois plus de données et de contenus sur Internet en un clic de manière complètement instantanée, mais en plus vous allez trouver des gens qui pensent comme vous, qui sont passionnés comme vous. Vous allez faire immédiatement communauté. Là où 15,20 ans en arrière vous auriez été peut-être découragé dans votre quête ésotérique, vous allez être aujourd'hui complètement galvanisé et enfermé dans une forme de bulle de filtres. C'est ce que l'on appelle le «biais de confirmation» : le fait que sur Internet vous trouvez ce que vous allez chercher. Si vous voulez trouver l'explication complotiste de n'importe quel événement, vous tapez le nom de l'événement et le mot « complot » à côté, et vous êtes à peu près certain de trouver un contenu qui répondra à vos attentes.

Emmanuel Taïeb : Donc cela va vous confirmer dans votre démarche initiale ou dans votre doute initial, qui ne sera pas levé ?

Rudy Reichstadt : Dans vos soupçons, absolument. Ça va nourrir votre soupçon, parce qu'on va vous apporter sur un plateau plusieurs arguments de nature à conforter ce que vous pensiez au préalable. Gérald Bronner parle de «millefeuilles argumentatif $\gg^{21}$, c'est-à-dire que l'on va remettre en cause la version communément acceptée des faits, la version stigmatisée comme « officielle », en présentant un ensemble d'arguments mis bout à bout, et des arguments parfois empruntés à des champs très diversifiés et très variés de la connaissance. Ce qui fait que vous ne pouvez pas avoir les compétences suffisantes pour démonter a priori chacun d'entre eux. Par conséquent, ça va produire sur vous un 
effet d'intimidation très fort, qui fait que vous allez vous dire que tout ne peut pas être faux, qu' « il n'y a pas de fumée sans feu ». Mission accomplie, puisque l'intérêt du conspirationnisme politique est évidemment d'instiller le doute.

Emmanuel Taïeb : Raphaël Josset, si on reste sur cette question d'Internet, vous évoquez les « conspirationnistes numériques », des conspirationnistes 2.0. Qui sont ces gens? Ce sont tous ces gens qui vont proposer des montages vidéo, qui vont spéculer, qui vont démonter l'information pour montrer que tout ça est manipulé ?

Raphaël Josset : L'émergence d'une nouvelle génération de « conspirationnistes numériques » est liée à l'avènement du web 2.0., cette nouvelle dynamique technologique qui arrive au milieu des années 2000, et qui ouvre les plateformes. Ça existait déjà un petit peu avec les blogs, les forums, mais ce web qui est basé sur le principe du « contenu généré par les utilisateurs »a été une révolution. C'était ça aussi le déferlement qui a largement contribué au développement de ces nouvelles tendances. Mais pour que cela prenne, il faut qu'il y ait une disposition mentale aussi. On peut tenir un discours, on peut avoir les dispositifs techniques pour le faire, mais pour que ça prenne à un moment donné, il faut qu'il y ait des gens qui adhèrent. Il faut qu'il y ait quelque chose qui colle complètement à ce discours-là, quelque chose qui mentalement entre dans un processus cognitif à ce moment-là. C'est lié à cette virtualité.

Les réseaux permettent la diffusion large de contenus « alternatifs » à la vitesse de la lumière. Mais c'est aussi ce qui fait qu'on se plonge dans les écrans, qu'on se plonge dans l'image, dans le virtuel, et c'est cette « virtualisation du monde » qui est aussi la cause de cette confusion entre le vrai et le faux. On ne sait plus à quoi se fier dans cet océan d'informations qui nous submerge, et finalement on cherche à retrouver du sens à travers un récit dont on dit qu'il avait disparu. C'était le symptôme de l'avènement de « la condition postmoderne » comme disait Jean-François Lyotard. Je pense qu'on a toujours besoin de se raccrocher à quelque chose, d'avoir des repères, d'avoir des récits et du sens, de la transcendance aussi. C'est un retour à un récit qui remet de la verticalité, de la transcendance divine, de la verticalité dans un monde de plus en plus horizontal, fluctuant, flottant, de plus en plus spectral.

Emmanuel Taïeb : Le site anti-conspirationniste ConspiHunter a fabriqué en 2016 un faux documentaire conspirationniste, signé d'un réalisateur virtuel Lionel Perrottin, qui défend l'idée que les États-Unis auraient implanté le sida à Cuba puis en Afrique, pour voir comment ce faux film va circuler sur les réseaux sociaux et éventuellement être repris et sans critique par les réseaux conspirationnistes même. Effectivement, c'est ce qui va se passer au-delà des espérances ?

Rudy Reichstadt : En l'occurrence, j'ai participé à cette initiative. Il s'agissait d'abord de faire une fausse vidéo complotiste, de la faire buzzer sur Internet, de la retirer ensuite puis de mettre le contre-poison qui est en fait le making of de cette vidéo, qui dure beaucoup plus longtemps, et de le projeter auprès des élèves. Je signale d'ailleurs que la vidéo a été rachetée par le réseau Canopé (ex-CNDP), par l'Éducation nationale. Elle est visible et accessible gratuitement. 
Emmanuel Taïeb : C'est devenu un outil pédagogique?

Rudy Reichstadt : C'est devenu un outil pédagogique. C'était conçu comme ça au départ. Il s'agissait de dire «on va écrire un scénario complotiste, on va en faire une vidéo, on va trouver sur Internet ce qui nous permet de nourrir cette théorie du complot, qui explique en gros que le sida a été créé par la CIA et implanté délibérément à Cuba, et ça expliquerait le blocus autour de Cuba». On s'est aperçu que ça avait pris et avait été repris sur deux plateformes conspirationnistes assez influentes, c'est-à-dire des sites qui sont dans le top 10 des sites conspirationnistes les plus fréquentés en langue française. Donc, ce n'est pas rien quand même. Ça a été repris comme si c'était un travail sérieux et de manière complètement a-critique. Ce qui permet quand même de souligner là l'asymétrie cognitive du complotisme. On a coutume de dire que les complotistes sont ceux qui doutent de tout, qui remettent toujours tout en cause. Or, ce n'est pas tout à fait exact. En fait, ils ont un doute très sélectif, à géométrie variable. Ils doutent de tout ce qui peut remettre en cause ce qu'ils appellent la « version officielle», mais ils acceptent le plus souvent sans critiques et avec beaucoup de crédulité tout ce qui va dans le sens de la théorie du complot. Ce qui pose quand même un problème.

Emmanuel Taïeb : En fait, ils ont repris ce film sans du tout le vérifier. Parce qu'au fond ça allait dans leur sens, même si c'était un « scoop » qui était proposé par le film. Ce qui montre visiblement qu'ils reprennent ce qui est de l'ordre de la destruction du système ou de la dénonciation anti-impérialiste, antiaméricaine sans aucune vérification de rigueur?

Rudy Reichstadt : C'est exactement ça, mais ça conforte leur représentation initiale, et c'est ça fondamentalement qu'il faut comprendre avec le complotisme.

Emmanuel Taïeb : Raphaël Josset, la nouveauté très forte du conspirationnisme 2.0 c'est la viralité, c'est-à-dire cette capacité d'utiliser la société en réseau, les réseaux sociaux, les réseaux Internet, pour se diffuser massivement et sortir d'une forme de marginalité dont Rudy Reichstadt parlait tout à l'heure. Vous diriez que c'est ça le fondement de la force du conspirationnisme 2.0 ?

Raphaël Josset : Ça ne marche pas sans dispositions mentales liées à des conditions objectives d'une époque qui font qu'il y a des gens qui sont réceptifs à ce genre de discours, à ce genre de virus, à ce genre de virus mental. On voit bien qu'il se diffuse depuis deux siècles. C'est exactement le même discours, on peut remonter à la Révolution française, à Augustin Barruel ${ }^{22}$. Le discours sur les Illuminati, il est déjà là, il existe depuis deux siècles, mais désormais ça se diffuse planétairement avec un nouveau média. Et en même temps il faut penser peut-être à la formule de McLuhan comme quoi « le médium est le message » : au-delà du contenu, il y a aussi la forme, le dispositif, le canal, la technique que l'on utilise, c'est le message premier et fondamental parce qu'il nous parle de la mutation de notre façon d'être au monde, de la façon dont il bouleverse nos manières de nous rapporter à l'espace et au temps. Et donc ça joue fondamentalement sur nos conditions, notre condition humaine, notre 
condition post-humaine bientôt. Il y a de nouveaux processus cognitifs qui sont liés à ça.

Après, on revient sur des choses très fondamentales, très archaïques, qui sont simplement le retour à une forme de pensée paranoïaque biaisée. J'aime bien le mot paranoïa si on ne le prend pas juste dans le sens de sentiment de persécution : « para », « noïa », c'est « l'esprit et la pensée », c'est le « para » de " parallèle », un mode de pensée parallèle. Quand des individus cherchent des théories critiques, pourquoi vont-ils vers des théories biaisées ? Il est difficile à expliquer aux gens que leur mode de penser n'est pas le bon. Tout ce qu'on peut faire par rapport à ça, c'est effectivement avoir une démarche pédagogique, expliquer aux gens, montrer, faire des documentaires, des choses comme ça.

J'ai vu le documentaire que nous évoquions à l'instant. Il est intéressant. Mais j'ai eu l'occasion de dire directement à Thomas Huchon, du site Spicee, qui est à l'origine du documentaire, que ce qui me posait problème dans ce documentaire, c'est qu'il y avait encore le montage un peu spectaculaire et sensationnaliste, et la dénomination ConspiHunter, " chasseur de conspis ». Je ne pense pas que ce soit la bonne démarche, parce que les gens qui sont considérés comme des conspirationnistes par d'autres vont s'enfermer dans ce rôle-là, et dans une posture de défiance et de dénégation. On ne fait que renforcer les gens dans leurs positions. Mais au-delà de ça, il y a d'autres choses à faire aussi : un traitement de fond sur la crise civilisationnelle, la crise existentielle et spirituelle. Ce n'est pas juste avec des arguments rationnels qu'on va réussir à montrer aux gens qu'ils se trompent.
Rudy Reichstadt : Sur le conspirationnisme 2.0 ou numérique, je crois qu'il ne faudrait pas commettre l'erreur de penser qu'il y a une barrière étanche entre ce qui se passe sur Internet et ce qui se passe dans le reste de la société. Il y a des allers-retours permanents entre les deux. En fait, les utilisateurs et les internautes sont des gens en chair et en os derrière leur écran d'ordinateur, et se nourrissent de ces choses-là aussi, et que ça a un impact direct sur notre société. On l'a vu aux États-Unis, lors des dernières élections américaines, où le vainqueur a fait un usage immodéré et assez largement irresponsable des théories du complot. Je ne dis pas que ça a présidé à son élection, mais en tout cas ça ne l'a pas empêché d'être élu.

En France aussi on a assisté à une forme de « trumpisation » de cette campagne présidentielle qui est absolument inédite. Lorsque vous avez des mots comme « oligarchie » qui circulent, on n'est pas loin de Charles Maurras, le fondateur de l'Action française royaliste, qui était dans le « nationalisme intégral », et évoquait quatre états confédérés, parlait des « quatre oligarchies ». C'étaient les protestants, les francs-maçons, les métèques et les juifs. L'extrême droite - et pas seulement elle - parlait d' « oligarchie ». Voir que ce vocable a réinvesti notre lexique politique contemporain, voir aussi bien Jean-Luc Mélenchon que Marine Le Pen l'utiliser, est une source d'inquiétude. Que des sociologues comme les époux Pinçon-Charlot parlent de complot oligarchique, par exemple, ça va assez loin. C'est une source d'inquiétude.

Après, je ne sais pas si on est effectivement dans l'ère post-idéologique. Les grandes structures 
et les grands récits des religions séculières, le marxisme en particulier, se sont effondrés. On ne raisonnait pas dans le marxisme en termes d' " oligarchie », de puissants d'un côté, d'élites financières ou que sais-je, et de l'autre, des petits et des opprimés. Le langage était celui d'une dialectique de classes. Il y a en fait quelque chose de très régressif dans ce conspirationnisme contemporain.

Emmanuel Taïeb : Lors des dernières campagnes électorales aux États-Unis et en France, il y a eu le développement inédit de ce que l'on appelle le «trolling » politique, c'est-à-dire le fait d'inonder volontairement les réseaux sociaux d'informations trafiquées ou fausses. Il y a eu une explosion des fake news, et on s'est rendu compte à ces occasions que les médias professionnels ont eu énormément de mal à désintoxiquer ou à lutter contre ces informations. Comment l'expliquez-vous ? Est-ce finalement le triomphe de l'idéologie sur la véracité de l'information ? Y a-t-il une demande de réalité alternative que les « fake news » viendraient combler?

Rudy Reichstadt : On commettrait une erreur en pensant qu'en démocratie on vote en faveur du discours le plus vrai, c'est-à-dire le plus conforme à la réalité des faits. On vote par conviction et souvent au nom d'une vérité surplombante supposée supérieure. À la rigueur le fait que Donald Trump ou Marine Le Pen, ou d'autres, ne disent pas des choses dans leur discours qui soient absolument conformes à la réalité des faits est presque secondaire. L'important, quand on est un électeur de Marine Le Pen par exemple, c'est que l'on soit d'accord avec ce qu'elle dit sur l'immigration, qu'on la crédite d'une volonté réelle de régler ce problème-là ou d'en découdre en tout cas. C'est identique avec Trump. Il est quand même frappant, lorsqu'on s'intéresse à leurs électeurs, qu'ils les créditent chacun de «parler vrai », ou de « tenir un discours de vérité ». Eux « parlent vrai », alors que par ailleurs - et pour Trump la démonstration a été faite pendant la campagne présidentielle - $70 \%$ des phrases que Donald Trump a pu prononcer étaient, au minimum, à demi vraies, au pire complètement fausses. Mais apparemment ce n'est pas ce qui compte pour ces électeurs.

Emmanuel Taïeb : Pour revenir sur cette question de la production d'infos, on a l'impression que désormais chaque individu peut spéculer chez lui sur le réel. On a des vidéos qui circulent où des gens filment leur télévision, et disent : « là on nous dit n'importe quoi », "là c'est faux ». Comment expliquer ce besoin de spéculer? Chacun est devenu producteur d'une interprétation du monde?

Raphaël Josset : J'ai le sentiment que tout le monde est devenu hyper politisé d'un seul coup. Avant, on avait un peu de mal à parler politique, ou c'est parce que j'avais 20 ans, j'étais plus jeune, que les gens autour de moi n'avaient pas trop envie d'en parler. Et maintenant j'ai l'impression que d'un seul coup tout le monde a un discours très tranché, très dur. Les gens se sentent floués. On est tellement baigné dans cette information qu'on ne sait pas si elle est vraie ou si elle est fausse. Tout est un peu fake, d'une manière générale. Parfois on peut vérifier des choses. Mais il y a la façon dont on enveloppe cette vérité, la façon dont on raconte les choses. 
Les auditeurs ne voient pas, mais ils peuvent imaginer que la bouteille là [posée dans le studio], nous la voyons de façon différente. Chacun de nous a un point de vue différent sur la chose. Pourtant elle est là, elle existe. Mais on pourrait la représenter, la décrire de manière différente chacun de nous. Tout est comme ça, rien n'est absolument vrai. En tout cas, c'est difficile d'en parler dans la représentation et dans le langage. On est toujours dans la représentation de toute façon. À partir de là, il y a un besoin de spéculation. Il y a un besoin de s'exprimer et souvent d'avoir un impact, une influence, et c'est quand on dit des choses un peu sensationnelles, étranges, et polémiques que ça fait réagir.

Emmanuel Taïeb : La diffusion du conspirationnisme est devenue assez forte et les pouvoirs publics s'en sont saisis notamment après les attentats de janvier 2015, en constatant que beaucoup de collégiens étaient finalement pétris de conspirationnisme, et qu'ils s'abreuvaient aux sources «alternatives » dont on parlait. C'est devenu un problème public. Pour rester sur l'inquiétude qu'on évoquait en début d'émission, est-ce qu'il n'est pas déjà trop tard pour inverser la tendance ? Comment fait-on pour lutter contre la vague conspirationniste, quand les médias sont vus comme incarnant le discours « officiel»?

Rudy Reichstadt : Si on renonce, il n'y a plus qu'à tirer l'échelle. S'agissant des plus jeunes, j'espère que rien n'est joué quand même. On joue avec ces choses-là aussi. L'adolescence c'est aussi l'âge de la transgression, où on remet en cause toute parole d'autorité, la parole adulte en général. Donc, rien n'est joué, je crois, à 15 ans.
Vous avez raison de souligner que les pouvoirs publics s'en sont emparés. Il y a une prise de conscience très nette, et qu'on peut dater du mois de janvier 2015. Dans le sillage des attentats de Charlie Hebdo, l'Éducation nationale, le président de la République, ont pris des positions très fortes pour la première fois. Pour parler explicitement de conspirationnisme désigné comme un problème public, que peut-on faire ? Je crois justement que les médias classiques et l'État ne peuvent pas tout faire. C'est vraiment un problème qui traverse toute la société. C'est aussi à la société civile d'y répondre, chacun selon son domaine de compétence, d'essayer de collaborer ou d'apporter dans l'espace public son expertise pour essayer de démythifier ces choses-là. 


\section{$\mathrm{N} \cdot \mathrm{O} \cdot \mathrm{T} \cdot \mathrm{E} \cdot \mathrm{S}$}

1. Matt Moore, «Shaq was apparently trolling everyone when he made his flat-Earth comments ", CBS Sports, 23/03/2017

https://www.cbssports.com/nba/news/shaq-wasapparently-trolling-everyone-when-he-made-his-flatearth-comments/ (consulté le 02/11/2017)

2. La chaine russe REN TV a récemment diffusé une émission "scientifique » prenant fait et cause pour les «théories » de la terre plate. Cf. Iegor Gran, «Scoop la terre est plate!", Charlie Hebdo, 25/10/2017. Sur les ressorts du conspirationnisme russe, cf. Stéphane François et Olivier Schmitt, "Le conspirationnisme dans la Russie contemporaine ", Diogène, "Les théories du complot aujourd'hui », 249-250, janvier-juin 2015.

3. Pierre-André Taguieff, Les "Protocoles des sages de Sion ». Faux et usages d'un faux, Berg international, 2004.

4. Pierre Birnbaum, Genèse du populisme. Le peuple et les gros, Paris, Pluriel, 2012 [1984].

5. Pierre-André Taguieff, La foire aux Illuminés. Esotérisme, théorie du complot, extrémisme, Paris, Mille et une nuits, 2005.

6. Eva Soteras, « Rockin' Squat : le conspirationnisme sur fond rythmique », Diogène, op. cit.

7. Véronique Campion-Vincent, La sociétéparano. Théories du complot, menaces et incertitudes, Paris, Payot, 2005.

8. Emmanuel Taïeb, " Logiques politiques du conspirationnisme. "Sociologie et sociétés, vol. 42, 2, automne 2010, p. 265-289.

$<$ doi:10.7202/045364ar>

9. Raphaël Josset, Complosphère. L'esprit conspirationniste à l'ère des réseaux, Paris, Lemieux éditeur, 2015.

10. <www.conspiracywatch.info>

11. Gilbert Durand, L'imagination symbolique, Paris, PUF, 2003 [1964].

12. Léon Poliakov, La Causalité diabolique. Essai sur l'origine des persécutions. Du joug mongol à la victoire de Lénine, Paris, Calmann-Lévy, 2006 [1980 \& 1985].

13. Raphaël Josset, Complosphère, op. cit., p. 11.

14. Richard Hofstadter, Le style paranö̈aque. Théories du complot et droite radicale en Amérique, Paris, François Bourin éditeur, 2012.

15. Emmanuel Taïeb, " "Repolitiser" la parole complotiste », Conspiracy Watch, 08/10/2013.

$<$ http://www.conspiracywatch.info/repolitiserla-parole-complotiste_a1125.html>

16. Joël Gombin, Conspiracy theories in France. Interim report, Counterpoint, 03/05/2013

<http:// counterpoint.uk.com/wp-content/ uploads/2013/05/Conspiracy-Theories-in-Franceinterim-report-3rd-May.pdf $>$

17. Norman Cohn, Les Fanatiques de l'Apocalypse. Millénaristes révolutionnaires et anarchistes mystiques au Moyen Age, Paris, Payot, 1983.

18. Norman Cohn, Histoire d'un mythe. La "conspiration" juive et les "Protocoles des sages de Sion », Paris, Gallimard, 1992.

19. Ernesto de Martino, Le monde magique, Paris, Institut d'édition Sanofi-Synthélabo, 1999.

20. Rudy Reichstadt, «Conspirationnisme : un état des lieux ", Note de la Fondation Jean Jaurès - Observatoire des radicalités politiques, 24/02/2015

<http://temps-reels.net/site/wp-content/ uploads/2015/04/2015-02-24_FJJ_conspirationnisme. pdf $>$

21. Gérald Bronner, La démocratie des crédules, Paris, PUF, 2013. On verra l'entretien avec G. Bronner dans ce numéro.

22. En 1798, dans ses Mémoires pour servir à l'bistoire du jacobinisme, l'abbé Barruel écrivait : «Dans cette Révolution française, tout jusqu'à ses forfaits les plus épowvantables, tout a été prévu, médité, combiné, résolu, statué : tout a été l'effet de la plus profonde scélératesse; puisque tout a été préparé, amené par des bommes qui avaient seuls le fil des conspirations longtemps ourdies 
dans des sociétés secrètes, et qui ont su choisir et hâter les moments propices aux complots. »

\section{$\mathrm{R} \cdot \mathrm{E} \cdot \mathrm{S} \cdot \mathrm{U} \cdot \mathrm{M} \cdot \mathrm{E}^{\prime}$}

Dans cet entretien, Raphaël Josset et Rudy Reichstadt reviennent sur l'imaginaire du conspirationnisme, et son idée théologique d'un affrontement entre le Bien et le Mal. Ils montrent que le développement des nouvelles technologies, et en particulier des réseaux sociaux, permettent la diffusion de discours hostiles à la science et à la parole « officielle» des politiques et des médias. Les sites conspirationnistes, qui se présentent comme des « agences de presse alternatives », relaient des « fake news » et des récits de complots sans les vérifier, pour des raisons politiques et idéologiques. Le succès des «théories » du complot implique cependant des croyances préalables, une attirance pour des théories critiques biaisées, et le triomphe du doute dans une société.

\footnotetext{
Abstract

In this interview, Raphaël Josset and Rudy Reichstadt focus on the imaginary of conspiracism, and its theological idea of a confrontation between Good and Evil. They show that the development of new technologies, especially the social networks, allow the broadcast of discourses that criticize science and "official" speeches of politicians and the media. Conspiracist websites, which claim to be "alternative press agencies", communicate fake news and conspiracy narratives, without any fact checking, for political and ideological reasons. Yet, the success of conspiracy "theories" implies preexisting beliefs, an attraction to biased critique theories, and the reign of doubt in a society.
} 